\title{
Herbig Ae/Be Stars in the Magellanic Bridge
}

\author{
Shogo Nishiyama ${ }^{1,2}$, Yasuaki Haba ${ }^{2}$, Daisuke $\mathrm{Kato}^{2}$, Daisuke Baba ${ }^{2}$, Hirofumi Hatano ${ }^{2}$, \\ Motohide Tamura $^{1}$, Yasushi Nakajima ${ }^{1}$, Akika Ishihara ${ }^{1,3}$, Tetsuya Nagata ${ }^{4}$, Koji \\ Sugitani $^{5}$, Noriyuki Matsunaga ${ }^{6}$, Hinako Fukushi ${ }^{6}$, Nobuhiko Kusakabe ${ }^{7}$, and Shuji Sato ${ }^{2}$
}

\begin{abstract}
We have found Herbig Ae/Be star candidates in the western region of the Magellanic Bridge. Using the near infrared camera SIRIUS and the $1.4 \mathrm{~m}$ telescope IRSF, we surveyed $\sim 3.0 \times 1.3\left(24^{\circ} \lesssim \alpha \lesssim 36^{\circ},-75^{\circ} .0 \lesssim \delta \lesssim-73^{\circ} .7\right)$ in the $J, H$, and $K_{S}$ bands. On the basis of colors and magnitudes, about $200 \mathrm{Herbig} \mathrm{Ae} / \mathrm{Be}$ star candidates are selected. Considering the contaminations by miscellaneous sources such as foreground stars and early-type dwarfs in the Magellanic Bridge, we estimate that about $80(\approx 40 \%)$ of the candidates are likely to be Herbig $\mathrm{Ae} / \mathrm{Be}$ stars. We also found one concentration of the candidates at the young star cluster NGC 796, strongly suggesting the existence of pre-main-sequence (PMS) stars in the Magellanic Bridge. This is the first detection of PMS star candidates in the Magellanic Bridge, and if they are genuine PMS stars, this could be direct evidence of recent star formation. However, the estimate of the number of Herbig $\mathrm{Ae} / \mathrm{Be}$ stars depends on the fraction of classical Be stars, and thus a more precise determination of the Be star fraction or observations to differentiate between the Herbig Ae/Be stars and classical Be stars are required.
\end{abstract}

Subject headings: stars: pre-main-sequence — stars: formation — infrared: stars - Magellanic Clouds

\footnotetext{
${ }^{1}$ National Astronomical Observatory of Japan, Mitaka, Tokyo 181-8588, Japan; shogo@optik.mtk.nao.ac.jp

${ }^{2}$ Department of Astrophysics, Nagoya University, Nagoya 464-8602, Japan

${ }^{3}$ Department of Earth and Planetary Science, School of Science, University of Tokyo, Bunkyo-ku, Tokyo 113-0033, Japan

${ }^{4}$ Department of Astronomy, Kyoto University, Kyoto 606-8502, Japan

${ }^{5}$ Graduate School of Natural Sciences, Nagoya City University, Nagoya 467-8501, Japan

${ }^{6}$ Institute of Astronomy, School of Science, University of Tokyo, Mitaka, Tokyo 181-0015, Japan

${ }^{7}$ Department of Astronomical Sciences, Graduate University for Advanced Studies (Sokendai), Mitaka, Tokyo 181-8588, Japan
} 


\section{INTRODUCTION}

The wing of the Small Magellanic Cloud (SMC) was detected by Shapley (1940), who described it as a large elliptical extension consisting of a cloud of blue stars. He also implied that there is a physical connection between the SMC and the Large Magellanic Cloud (LMC), since the wing extends from the center of the SMC in the direction of the LMC. A number of H I surveys (e.g., Hindman, Kerr, \& McGee 1963; Muller et al. 2003a) have since revealed a continuous bridge of gas between the SMC and LMC, now known as the Magellanic Bridge (MB). The optical "stellar bridge" was found by Irwin, Demers, \& Kunkel (1990) between the wing and the western halo of the LMC, showing that the SMC wing is the brightest section of the stellar link between them. The wing is the richest region of blue stars (Irwin. Demers, \& Kunkel 1990), clusters/associations (Bica \& Schmitt 1995), and H I gas (Muller et al. 2003a) in the MB, and is thus expected to be the most active in star formation.

The MB is the closest bridge $(60 \mathrm{kpc}$, assuming that its distance is the same as that to the SMC; e.g., Harries, Hilditch, \& Howarth 2003) among a number of known intergalactic bridges situated in groups of galaxies. The mechanism responsible for the formation of the MB is widely considered to be the gravitational influence of the LMC (e.g., Gardiner, Sawa \& Fujimoto 1994). Because of tidal interactions, star formation is likely to be very active in the bridges, and the details of these interactions can be investigated through the study of young stars, such as OB and pre-main-sequence (PMS) stars. The MB can also provide us with insights into the evolutionary development of dwarf galaxies as well as the role of external dynamical interactions in stimulating star formation. The relative importance of the star formation processes due to external and internal disturbances to the SMC is one of the key questions yet to be properly answered.

Another important aspect of observations is that stars in the MB can provide valuable clues for understanding star formation processes in a low metallicity environment. The very low metallicity of the MB was found to be $\sim 0.5$ dex lower than that of the SMC (Rolleston et al. 1999), which itself is much lower than our Galaxy. De Wit et al. (2003) discussed properties of PMS (Herbig Ae/Be) stars in the SMC and suggested that such stars have luminosities generally higher than those of the same spectral type in our Galaxy, indicating a faster proto-stellar mass accretion rate at lower metallicity (see also; Beaulieu et al. 1996, 2001; Lamers et al. 1999; Panagia et al. 2000; de Wit et al. 2002, 2005). Because of their vicinity, the PMS stars in the MB offer a good opportunity to study in detail the formation and evolution of individual young stars in different environments.

Previous studies of the star formation processes in the MB have mainly concentrated on stellar populations at optical wavelengths (e.g., Westerlund \& Glaspey 1971; Demers \& Battinelli 
1998) and CO content (Muller et al. 2003b; Mizuno et al. 2006). In this paper, we present the result of our near infrared $\left(J, H, K_{S}\right)$ survey of the western region in the MB (the wing of the SMC). The limiting magnitudes of the survey are $\sim 2-3$ mag deeper than the Two Micron All Sky Survey (2MASS), and this difference is critical to detecting intermediate-mass young stellar objects (i.e., Herbig Ae/Be stars) at the distance of the Magellanic Clouds and the MB. The age of the Herbig Ae/Be stars we can detect is less than $\sim 10^{6} \mathrm{yr}$, the youngest stellar population ever found in the MB. Our detection will also make it possible to understand star formation processes in a low metallicity environment in the region far from the center of the SMC.

\section{OBSERVATIONS AND DATA REDUCTION}

Our observations were conducted in 2004 August and 2005 March, July-August using the near-infrared camera SIRIUS (Simultaneous Infrared Imager for Unbiased Survey; Nagashima et al. 1999; Nagavama et al. 2003) on the IRSF (Infrared Survey Facility) telescope. IRSF is a $1.4 \mathrm{~m}$ telescope constructed and operated by Nagoya University and SAAO (South African Astronomical Observatory) at Sutherland, South Africa. The SIRIUS camera can provide $J(1.25 \mu \mathrm{m}), H(1.63 \mu \mathrm{m})$, and $K_{S}(2.14 \mu \mathrm{m})$ images simultaneously, with a field of view of $7 ! 7 \times 7 ! 7$ and a pixel scale of 0 '! 45 .

The total area covered by our survey is $\sim 4 \operatorname{deg}^{2}$ (324 fields) over $1^{\mathrm{h}} 36^{\mathrm{m}} \lesssim \mathrm{RA}(\mathrm{J} 2000.0) \lesssim$ $2^{\mathrm{h}} 24^{\mathrm{m}}$ and $-75.0 \lesssim \operatorname{Dec}(\mathrm{J} 2000.0) \lesssim-73^{\circ} .7$ (Fig. 1). We observed only on photometric nights, and the typical seeing was 1".1 FWHM in the $H$ band. A single image comprises 10 dithered $30 \mathrm{~s}$ exposures.

Data reduction was carried out with the IRAF (Image Reduction and Analysis Facility) 1 software package. Images were prereduced following the standard procedures of near-infrared arrays (dark frame subtraction, flat-fielding, and sky subtraction). Photometry, including point-spread function (PSF) fitting, was carried out with the DAOPHOT package (Stetson 1987). We used the DAOFIND task to identify point sources, and the sources were then utilized for PSF-fitting photometry in the ALLSTAR task. Each image was calibrated with the standard stars 9103, 9106, and 9109 (Persson et al. 1998). The averages of the $10 \sigma$ limiting magnitudes were $J=19.0, H=18.0$, and $K_{S}=17.2$. The averages of the zero-point uncertainties were about $0.03,0.03$, and $0.04 \mathrm{mag}$ in the $J, H$ and $K_{S}$ bands, respectively.

\footnotetext{
${ }^{1}$ IRAF is distributed by the National Optical Astronomy Observatory, which is operated by the Association of Universities for Research in Astronomy, Inc., under cooperative agreement with the National Science Foundation.
} 
We also confirmed that the magnitude difference between our data and 2MASS is 0.02-0.04 mag in the three bands. This data set is the preliminary version of "The IRSF Magellanic Clouds Point Source Catalog"; a more detailed description of our analysis is given in D. Kato et al. (2007, in preparation).

\section{SELECTION OF HERBIG Ae/Be STARS}

In our fields, a total of 49,865, 34,964, and 22,094 stars are detected in the $J, H$ and $K_{S}$ bands, respectively, within the $10 \sigma$ limiting magnitude. To find PMS stars with infrared excess, we made a $J-H$ versus $H-K_{S}$ color-color (CC) diagram (Fig. 2). Only 20,261 stars detected in all three bands with photometric errors $\sigma_{\text {phot }} \leq 0.1$ mag are plotted. The $\mathrm{CC}$ diagram of the MB shows three distinct features. First, most $(\sim 94 \%)$ of the stars are distributed around dwarf and giant loci of $\mathrm{F}$ to $\mathrm{M}$ spectral types $(0.2 \lesssim J-H \lesssim 0.8$, $\left.-0.2 \lesssim H-K_{S} \lesssim 0.5\right)$. They are dominated by foreground sources, and their classification into foreground and Bridge sources is not straightforward. Second, some sources $(\sim 3 \%$, $\sim 800$ ) are located at $0.5 \lesssim J-H \lesssim 1.2$ and $0.5 \lesssim H-K_{S} \lesssim 1.0$. Most of them are fainter than $15.0 \mathrm{mag}$ in the $J$ band. There could be three populations in this area: group I/II Herbig Ae/Be stars (Hillenbrand et al. 1992), AGB stars, and predominantly galaxies (cf. Nakajima et al. 2005). Third, we can find some sources at $J-H \lesssim 0.2$ and $H-K_{S} \lesssim 0.4$. They are located on and rightward of the dwarf locus, indicating that they are normal early type (OB) dwarfs and stars with infrared excess.

Herbig Ae/Be stars in our Galaxy show strong excess radiation at infrared wavelengths. This excess is interpreted in terms of circumstellar dust emission with a wide range in temperatures. For our sources redder than $J-H=0.2$, selection of the Herbig Ae/Be stars is complicated due to contamination by dwarfs/giants and background galaxies (see Fig. 2). Since the Herbig Ae/Be stars in the LMC and SMC have a bluer color than those in our Galaxy (e.g., de Wit et al. 2003, 2005), we searched the region bluer than $J-H=0.2$, where stars with small infrared excess are located, 2 for the Herbig Ae/Be stars.

We selected Herbig Ae/Be candidates with the following criteria: (1) $J-H \leq 0.2$, (2) $J \geq 13.4$, (3) more than 0.1 mag apart from the OB locus, and (4) located under the

\footnotetext{
${ }^{2}$ In this analysis, we regard the interstellar extinction as negligible. The largest $\mathrm{H}$ I column density in our region is $\sim 1.5 \times 10^{21} \mathrm{~cm}^{-2}$, corresponding to $0.79 \mathrm{mag}$ in the $V$ band and 0.064 mag in the $K$ band for solar metallicity $\left(N_{H} / A_{V}=1.9 \times 10^{21} \mathrm{~cm}^{-2} \mathrm{mag}^{-1}\right.$ [Bohlin, Savage, \& Drake 1978], $A_{K}=0.081 \times A_{V}$ [van de Hulst 1946]). Moreover, the metallicity of the MB is much lower than the solar one, hence extinction is expected to be much smaller than 0.064 mag in the $K$ band.
} 
line $J-H=4.2 \times\left(H-K_{S}\right)-0.23$. Stars with $J-H<-0.1$ and $H-K_{S}>0.4$ were also excluded. The first criterion is, as discussed above, to exclude Galactic late-type (later than F5) dwarfs/giants and galaxies. Early type stars in our Galaxy and supergiants in the $\mathrm{MB}$ are brighter than $\mathrm{O} 3 \mathrm{~V}$ stars in the $\mathrm{MB}$, and thus the second criterion was imposed to exclude such stars. With the third one, the OB dwarfs were rejected (see circles in Fig. 2).

The fourth criterion is imposed to find stars with infrared excess. Fig. 3 shows a histogram of the distances $d_{\text {locus }}$ from the dwarf locus at $0.0 \leq J-H \leq 0.2$, for the sources in the area "A" shown in Fig. 2, The locus in the area corresponds to A0-F5 dwarfs, and we assume that the locus is a linear line with $J-H=4.2 \times\left(H-K_{S}\right)-0.02$. We also assume that sources at $d_{\text {locus }}<0$ (the left side of the locus in the area A) are AF dwarfs, and those at $d_{\text {locus }}>0$ are AF dwarfs plus Herbig Ae/Be stars with the infrared excess. The AF dwarfs are expected to show a Gaussian distribution, $N\left(d_{\text {locus }}\right)=N_{0} \exp \left(-d_{\text {locus }}^{2} / 2 \sigma^{2}\right)$. The Gaussian distribution of AF dwarfs determined by fitting the histogram at $d_{\text {locus }}<0$ is shown in Fig. 3. The standard deviation of the fitting is $\sigma=0.057 \mathrm{mag}$ and $N_{0}=23.6$. We assume that the sources with $d_{\text {locus }} \geq 0.057 \mathrm{mag}=1 \sigma$ have infrared excess.

As a result, we found 203 Herbig Ae/Be star candidates (Table 1). Their spatial distribution is shown in Fig. 1. A concentration of the candidates associated with the star cluster NGC 796 is found at $01^{\mathrm{h}} 56^{\mathrm{m}}$ and $-74^{\circ} 13^{\prime}$ (shown by an open circle in Fig. 1), but we cannot find any other concentrations. The candidates are also plotted on the CC diagram (Fig. 2, crosses).

\section{DISCUSSION}

\subsection{Contamination by Miscellaneous Sources}

There could be three populations in the region where our Herbig Ae/Be candidates are distributed (shown by crosses in Fig. 2): Herbig Ae/Be stars, classical Be (CBe) stars, and dwarfs of O to F5 spectral types. To confirm whether Herbig Ae/Be stars exist in the MB or not, the number of the latter two populations contaminating the region should be estimated.

The contamination by AF (A0 to F5) dwarfs can be estimated by using the histogram shown in Fig. 3, As mentioned above, the distribution of distance from the AF dwarf locus can be given by a Gaussian function, and thus the number of AF dwarfs distributed at $d_{\text {locus }} \geq 0.057$ is calculated to be $\approx 25$.

Since OB dwarfs are distributed around the locus with an observational uncertainty, we can estimate how many OB dwarfs are contaminating the region of stars with the infrared 
excess. Among the sources detected in the three bands with $\sigma_{\text {phot }} \leq 0.1$ mag, about $26 \%$ have an uncertainty $\sqrt{\sigma_{J-H}^{2}+\sigma_{H-K_{S}}^{2}}>0.1 \mathrm{mag}$ (Fig. 4). This means that the number of OB dwarfs distributed outside of the 0.1 mag radius error circles (see, Fig. 2) is less than $26 \%$ of the number of those within the error circles (333 stars). Half (right side) of the outside stars are located in the region of stars with infrared excess, and thus contamination by OB dwarfs is less than $13 \%$.

CBe stars are B-type stars that exhibit line emission over the photospheric spectrum. The observational characteristics of CBe stars and Herbig Ae/Be stars are very similar, making it a very difficult task to differentiate between the two types at near-infrared wavelengths.

In the SMC and LMC fields, the mean number ratio of CBe stars to early B-type stars 3 is estimated to be $\sim 20 \%$ (Keller. Wood. \& Bessel 1999). From the 2MASS JHK magnitudes of Galactic CBe stars (Zhang. Chen, \& Yang 2005), we derived the fraction of CBe stars in the error circles discussed above (see also Fig. 2) and those selected as Herbig Ae/Be stars with the criteria from Sec. 3 to be $47 \%$ and $48 \%$, respectively, and thus we can estimate the number of contamination by $\mathrm{CBe}$ and $\mathrm{OB}$ stars as follows. The number of stars in the error circles, 333, is the sum of $74 \%$ of OB dwarfs and $47 \%$ of CBe stars $\left(0.74 N_{\mathrm{OB}}+0.47 N_{\mathrm{CBe}}=333\right.$, where $N_{\mathrm{OB}}$ and $N_{\mathrm{CBe}}$ are the number of OB and CBe stars, respectively). The result of Keller, Wood, \& Bessel (1999) gives $4 N_{\mathrm{CBe}}=N_{\mathrm{OB}}$, and thus we obtain $N_{\mathrm{CBe}} \approx 97$ and $N_{\mathrm{OB}} \approx 388$. Therefore, the number of contaminating sources is $0.48 N_{\mathrm{CBe}}+0.13 N_{\mathrm{OB}}+25$ (AFstars) $\approx 47+50+25=122$, suggesting that about $60 \%$ of the 203 Herbig Ae/Be candidates could be contaminated by the AF and OB dwarfs, and the CBe stars. Resultantly, we conclude that 81 of them are Herbig Ae/Be stars after eliminating the possible contaminating sources.

However, the frequency of the CBe stars among B-type stars is still controversial. By using bright field stars in our Galaxy, Zorec \& Briot (1997) reported that as many as onethird of early B (B0 to B4) stars show the Be phenomenon. If we took their frequency, a contamination of 151 stars would be found. On the other hand, in the sample of Galactic open clusters, McSwain \& Gies (2005) showed that Be stars comprise only $7.4 \%$ and 4.7 $\%$ of the evolved and early-type B stars, respectively. This could lead to the estimate that about half of the sources with infrared excess are Herbig Ae/Be stars. Hence, a more precise determination of the CBe frequency or observations to differentiate between the Herbig

\footnotetext{
${ }^{3}$ Six clusters used by Keller, Wood, \& Bessel (1999) are not so young (10-30 Myr) that Herbig Ae/Be stars are not included in their samples of Be stars.

${ }^{4}$ Following Zhang, Chen, \& Yang (2005), we regard all the stars in their list as classical Be stars, although there are a few Herbig Ae/Be stars (see their Sec. 2).
} 
Ae/Be stars and CBe stars are required to decide how many stars have been formed in the MB.

\subsection{NGC 796}

NGC 796 (Fig. 5) is one of the youngest clusters in the SMC-Bridge region, and its age is estimated to be less than $\sim 10 \mathrm{Myr}$ from the comparison of integrated spectra with those of template clusters (Ahumada et al. 2002). This is the only association with an IRAS counterpart in the inter-cloud region (Grondin \& Demers 1993). We have found six Herbig Ae/Be candidates in the $\sim 30^{\prime \prime}$ radius centered at NGC 796 (open circles in Fig. 5). Five OB star candidates (the stars near the dwarf locus of spectral types O-B on the CC diagram) are also distributed in the same radius (filled squares in Fig. 51).

As mentioned above, the CBe stars could be located at the infrared-excess area on the CC diagram; however, clusters younger than 10 Myr are almost completely lacking CBe stars (Fabregat \& Torrejón 2000; McSwain \& Gies 2005). Considering the age of NGC 796, most of the stars with infrared excess are likely Herbig Ae/Be stars. This fact further suggests that our criteria for selecting the Herbig Ae/Be stars are appropriate. The IRAS detection sensitivity for protostars at the distance of the MB (single protostars with masses $\gtrsim 10-20 \mathrm{M}_{\odot}$ or an association containing a few less massive stars, Grondin \& Demers 1993) also indicates that NGC 796 is a cluster of Herbig Ae/Be stars. We found no other strong concentrations of Herbig Ae/Be candidates in our survey region.

\subsection{Herbig Ae/Be Stars in the Magellanic Bridge}

Higher-mass PMS stars are not expected to be visible before they reach the zero-age main sequence due to obscuration by circumstellar dust envelopes/disks, but the visibility may depend on environmental conditions such as metallicity. For example, Beaulieu et al. (1996) have found Herbig Ae/Be candidates in the LMC with luminosities systematically higher than observed in our Galaxy. The candidates are located well above the birthline of Palla \& Stahler (1993) for an accretion rate of $10^{-5} \mathrm{M}_{\odot} \mathrm{yr}^{-1}$ in the HR diagram. Beaulieu et al. (1996) then concluded that their location in the HR diagram indicates a lower circumstellar extinction due to low metallicity, or a prevailing accretion rate of about

$10^{-4} \mathrm{M}_{\odot} \mathrm{yr}^{-1}, 10$ times higher than for Galactic stars of the same luminosity. Panagia et al. (2000) suggested a higher accretion rate for LMC low-mass PMS stars as well, and recently a statistically significant excess of the accretion rate $(\sim 30$ times more than that for Galactic 
stars at a comparable age) was found for low-mass PMS stars in the LMC (Romaniello et al. 2004). However, the location of the birthline could also be controlled by the deuterium mass fraction and stellar opacity, which are modified by changing the metallicity (Bernasconi 1996).

Our result suggests that the environmental effect of low metallicity can also be seen for the PMS stars in the MB. Fig. [6 shows the location of the Herbig Ae/Be candidates in the color magnitude (CM) diagram (crosses). Assuming that our candidates are the group III Herbig Ae/Be stars defined by Hillenbrand et al. (1992), the $J$ band magnitudes show that the candidates have masses in the range of $\sim 10$ to more than $30 \mathrm{M}_{\odot}$ (see horizontal dashed lines in Fig. 6). If they are members of the group $\mathrm{I} / \mathrm{II}$, the mass range is $\sim 3$ to more than $20 \mathrm{M}_{\odot}$ (horizontal dotted lines in Fig. 6). The Herbig Ae/Be candidates found in the LMC (filled triangles, de Wit et al. 2005) and in the SMC (filled squares, de Wit et al. 2003) are also plotted in Fig. 6. They are up to 10 times as luminous as the Galactic Herbig Ae/Be stars in the same temperature range (see, e.g., de Wit et al. 2003, Sec. 9.2 and Fig. 18), and both our candidates and theirs are located in a region where a few Galactic Herbig Ae/Be stars are found 5 The location of our candidates in the CM diagram thus implies that the formation process of the Herbig Ae/Be stars in the MB, as well as the LMC and SMC, is different from that in our Galaxy.

The MB is believed to have been generated by the recent collision of the Magellanic Clouds about 200 Myr ago (Gardiner, Sawa \& Fujimoto 1994). The existence of the Herbig Ae/Be stars, associations and other young massive stars ( $<20$ Myr, e.g., Demers \& Battinelli 1998, Rolleston et al. 1999) indicates that star formation is still occurring in the MB.

In the $\mathrm{MB}$, we found $\sim 80 \mathrm{Herbig}$ Ae/Be stars, whose smallest masses are about $3 \mathrm{M}_{\odot}$ (group I/II) and $10 \mathrm{M}_{\odot}$ (group III) (see, Fig. 6). According to the calculation by Bernasconi \& Maeder (1996), contraction times deduced for 3 and $9 \mathrm{M}_{\odot}$ stars at $Z=0.001$ are $\approx 1.7$ and $0.2 \mathrm{Myr}$, respectively. We can thus estimate, to the first order, the lower limit of the star formation rate in the MB in the last megayear as $\sim 1.4 \times 10^{-4} \mathrm{M}_{\odot} \mathrm{yr}^{-1}$ for group I/II. This rate is comparable to $\sim 4 \times 10^{-4} \mathrm{M}_{\odot} \mathrm{yr}^{-1}$, derived from the observation of ${ }^{12} \mathrm{CO}(1-0)$ emission (Mizuno et al. 2006).

How long the MB will survive as an independent system is as yet uncertain. The gas in the MB shows low velocities in the LMC-standard-of-rest frame, indicating that the gas will certainly be absorbed into the Magellanic Clouds (Brüns et al. 2005). However, more than

\footnotetext{
${ }^{5}$ We have adopted a distance to the MB of $60 \mathrm{kpc}$. If we adopted $50 \mathrm{kpc}$, which is the distance to the LMC, the stars we detected would be fainter by only 0.4 mag, but the Herbig Ae/Be candidates would still be brighter than the Galactic average.
} 
$10^{5} \mathrm{M}_{\odot}$ stars could be formed in a few gigayears (Yoshizawa \& Noguchi 2003) if it keeps its current star formation rate. This implies that the MB has the potential to evolve into a dwarf galaxy.

\section{CONCLUSION}

We have conducted a near infrared $\left(J, H, K_{S}\right)$ deep survey of the western region of the Magellanic Bridge (the wing of the SMC). Using color-color and color-magnitude diagrams, we have found $\sim 200$ Herbig Ae/Be star candidates. Considering contamination by other sources, we infer that about $40 \%$ of them are Herbig Ae/Be stars in the Magellanic Bridge. We have also found six Herbig Ae/Be star and five OB star candidates in NGC 796, showing the existence of a very young cluster in the region. The location of the Herbig Ae/Be candidates in the color-magnitude diagram indicates that they are more luminous than the Galactic ones.

We are grateful to Eric Muller for kindly providing the H I data of the Magellanic Bridge. We would like to thank Haruyuki Okuda, Norikazu Mizuno, and Akiko Kawamura for their helpful comments. We also thank the staff at the South African Astronomical Observatory (SAAO) for their support during our observations. The IRSF/SIRIUS project was initiated and supported by Nagoya University, the National Astronomical Observatory of Japan in collaboration with the SAAO under Grants-in-Aid for Scientific Research No.10147207, No.10147214, No.13573001, and No.15340061 of the Ministry of Education, Culture, Sports, Science and Technology (MEXT) of Japan. This work was also supported in part by the Grants-in-Aid for the 21st Century COE "The Origin of the Universe and Matter: Physical Elucidation of the Cosmic History" from the MEXT of Japan. 
Table 1. Catalog of Herbig Ae/Be Star Candidates in the Magellanic Bridge

\begin{tabular}{rrrrrccccc}
\hline \hline ID & R.A. (J2000.0) & Dec. (J2000.0) & $J$ & $\sigma_{J}$ & $H$ & $\sigma_{H}$ & $K_{S}$ & $\sigma_{K_{S}}$ \\
\hline 1 & 013748.37 & -734742.2 & 16.18 & 0.02 & 16.04 & 0.03 & 15.80 & 0.04 \\
2 & 013813.75 & -734808.9 & 16.35 & 0.02 & 16.31 & 0.03 & 16.12 & 0.04 \\
3 & 013815.48 & -740327.7 & 16.62 & 0.03 & 16.47 & 0.05 & 16.31 & 0.06 \\
4 & 013905.58 & -742747.0 & 16.79 & 0.03 & 16.63 & 0.06 & 16.26 & 0.08 \\
5 & 013915.82 & -741532.3 & 15.91 & 0.02 & 15.74 & 0.09 & 15.54 & 0.02 \\
6 & 013926.63 & -735802.9 & 16.22 & 0.02 & 16.15 & 0.02 & 16.01 & 0.04 \\
7 & 013934.99 & -740639.9 & 13.56 & 0.03 & 13.47 & 0.02 & 13.23 & 0.06 \\
8 & 014005.62 & -741340.8 & 14.19 & 0.02 & 14.20 & 0.03 & 13.83 & 0.03 \\
9 & 014029.37 & -734328.0 & 15.51 & 0.02 & 15.36 & 0.02 & 15.17 & 0.03 \\
10 & 014039.68 & -743243.5 & 17.03 & 0.04 & 16.89 & 0.06 & 16.65 & 0.09 \\
11 & 014042.83 & -735857.0 & 17.27 & 0.03 & 17.15 & 0.05 & 16.92 & 0.10 \\
12 & 014050.80 & -741030.9 & 15.16 & 0.02 & 15.03 & 0.02 & 14.80 & 0.02 \\
13 & 014142.49 & -735528.8 & 15.53 & 0.01 & 15.42 & 0.02 & 15.20 & 0.03 \\
14 & 014221.87 & -743014.0 & 16.69 & 0.02 & 16.63 & 0.05 & 16.47 & 0.09 \\
15 & 014226.35 & -741432.2 & 13.60 & 0.01 & 13.52 & 0.07 & 13.25 & 0.02 \\
16 & 014229.18 & -745046.8 & 15.05 & 0.01 & 14.92 & 0.02 & 14.76 & 0.02 \\
17 & 014233.64 & -735156.9 & 15.46 & 0.02 & 15.41 & 0.02 & 15.29 & 0.04 \\
18 & 014234.35 & -750033.8 & 17.18 & 0.02 & 16.99 & 0.04 & 16.81 & 0.08 \\
19 & 014309.84 & -741800.1 & 16.09 & 0.02 & 15.98 & 0.02 & 15.88 & 0.04 \\
20 & 014311.35 & -742350.2 & 16.14 & 0.02 & 16.05 & 0.02 & 15.86 & 0.04 \\
21 & 014335.61 & -734212.7 & 16.52 & 0.03 & 16.45 & 0.02 & 16.36 & 0.05 \\
22 & 014339.22 & -733931.6 & 17.56 & 0.04 & 17.49 & 0.06 & 17.32 & 0.09 \\
23 & 014343.00 & -740627.5 & 17.18 & 0.03 & 17.25 & 0.05 & 17.07 & 0.07 \\
24 & 014344.74 & -734656.4 & 14.98 & 0.02 & 14.89 & 0.02 & 14.77 & 0.03 \\
25 & 014359.29 & -744159.5 & 13.92 & 0.01 & 13.88 & 0.01 & 13.72 & 0.02 \\
26 & 014415.29 & -744054.1 & 16.24 & 0.02 & 16.17 & 0.02 & 16.06 & 0.04 \\
27 & 014447.42 & -744300.4 & 16.15 & 0.01 & 16.01 & 0.02 & 15.88 & 0.04 \\
28 & 014448.28 & -742822.3 & 14.95 & 0.01 & 14.93 & 0.09 & 14.80 & 0.02 \\
29 & 014453.65 & -743336.2 & 13.90 & 0.04 & 13.83 & 0.05 & 13.47 & 0.02 \\
30 & 014504.09 & -744418.2 & 15.75 & 0.01 & 15.64 & 0.02 & 15.53 & 0.03 \\
31 & 014505.01 & -735920.1 & 15.07 & 0.01 & 14.97 & 0.01 & 14.68 & 0.02
\end{tabular}


Table 1-Continued

\begin{tabular}{ccccccccc}
\hline \hline ID & R.A. (J2000.0) & Dec. (J2000.0) & $J$ & $\sigma_{J}$ & $H$ & $\sigma_{H}$ & $K_{S}$ & $\sigma_{K_{S}}$ \\
\hline 32 & 014507.23 & -741832.5 & 16.80 & 0.02 & 16.74 & 0.03 & 16.66 & 0.06 \\
33 & 014510.67 & -744326.7 & 14.61 & 0.01 & 14.56 & 0.01 & 14.47 & 0.02 \\
34 & 014525.11 & -742106.3 & 13.61 & 0.01 & 13.53 & 0.01 & 13.44 & 0.02 \\
35 & 014535.89 & -734103.2 & 15.92 & 0.02 & 15.76 & 0.02 & 15.49 & 0.03 \\
36 & 014543.78 & -734403.1 & 15.99 & 0.02 & 15.86 & 0.02 & 15.63 & 0.03 \\
37 & 014547.08 & -743153.2 & 16.34 & 0.03 & 16.45 & 0.03 & 16.30 & 0.06 \\
38 & 014611.29 & -734043.7 & 17.82 & 0.03 & 17.70 & 0.06 & 17.31 & 0.10 \\
39 & 014618.17 & -743337.4 & 16.53 & 0.02 & 16.38 & 0.04 & 16.22 & 0.06 \\
40 & 014639.84 & -744419.6 & 14.06 & 0.07 & 13.94 & 0.06 & 13.66 & 0.09 \\
41 & 014715.78 & -734009.3 & 16.25 & 0.02 & 16.20 & 0.02 & 16.09 & 0.04 \\
42 & 014721.50 & -745443.2 & 15.17 & 0.01 & 15.00 & 0.08 & 14.75 & 0.02 \\
43 & 014744.04 & -742252.0 & 17.11 & 0.03 & 17.09 & 0.05 & 16.94 & 0.09 \\
44 & 014810.27 & -735845.7 & 15.66 & 0.02 & 15.54 & 0.02 & 15.34 & 0.04 \\
45 & 014819.98 & -735550.0 & 15.92 & 0.01 & 15.85 & 0.02 & 15.65 & 0.04 \\
46 & 014911.89 & -735931.3 & 14.86 & 0.02 & 14.79 & 0.02 & 14.60 & 0.02 \\
47 & 014918.59 & -743753.8 & 15.79 & 0.02 & 15.75 & 0.02 & 15.62 & 0.04 \\
48 & 014929.37 & -743522.6 & 15.78 & 0.02 & 15.75 & 0.02 & 15.62 & 0.03 \\
49 & 014936.72 & -742838.1 & 14.39 & 0.02 & 14.34 & 0.06 & 14.18 & 0.06 \\
50 & 014954.88 & -743034.1 & 16.68 & 0.03 & 16.62 & 0.05 & 16.51 & 0.09 \\
51 & 015001.41 & -733944.2 & 14.86 & 0.07 & 14.67 & 0.02 & 14.38 & 0.02 \\
52 & 015020.13 & -735520.5 & 16.23 & 0.02 & 16.22 & 0.02 & 16.05 & 0.04 \\
53 & 015123.50 & -742637.1 & 15.76 & 0.02 & 15.69 & 0.02 & 15.40 & 0.03 \\
54 & 015127.42 & -743106.4 & 16.68 & 0.02 & 16.63 & 0.03 & 16.47 & 0.07 \\
55 & 015132.68 & -742550.1 & 17.15 & 0.02 & 17.19 & 0.07 & 17.03 & 0.08 \\
56 & 015138.41 & -743126.3 & 15.34 & 0.06 & 15.19 & 0.04 & 15.07 & 0.08 \\
57 & 015148.02 & -740426.7 & 15.88 & 0.02 & 15.83 & 0.03 & 15.57 & 0.04 \\
58 & 015156.94 & -743026.9 & 17.00 & 0.02 & 17.00 & 0.09 & 16.90 & 0.09 \\
59 & 015212.68 & -743621.9 & 16.67 & 0.03 & 16.53 & 0.03 & 16.34 & 0.06 \\
60 & 015213.97 & -734503.6 & 16.50 & 0.02 & 16.37 & 0.02 & 16.21 & 0.05 \\
61 & 015219.41 & -740417.4 & 16.88 & 0.03 & 16.83 & 0.05 & 16.64 & 0.09 \\
62 & 015219.69 & -743248.1 & 17.23 & 0.03 & 17.33 & 0.05 & 17.23 & 0.10
\end{tabular}


Table 1-Continued

\begin{tabular}{ccccccccc}
\hline \hline ID & R.A. (J2000.0) & Dec. (J2000.0) & $J$ & $\sigma_{J}$ & $H$ & $\sigma_{H}$ & $K_{S}$ & $\sigma_{K_{S}}$ \\
\hline 63 & 015228.27 & -742040.8 & 16.51 & 0.02 & 16.57 & 0.03 & 16.42 & 0.05 \\
64 & 015238.38 & -743852.2 & 14.83 & 0.02 & 14.76 & 0.01 & 14.62 & 0.03 \\
65 & 015241.21 & -742922.8 & 16.96 & 0.02 & 17.00 & 0.04 & 16.76 & 0.09 \\
66 & 015253.74 & -741948.3 & 17.07 & 0.02 & 17.12 & 0.04 & 17.01 & 0.09 \\
67 & 015255.76 & -740756.3 & 17.07 & 0.02 & 17.15 & 0.04 & 17.01 & 0.10 \\
68 & 015307.43 & -744254.8 & 16.98 & 0.02 & 16.93 & 0.04 & 16.80 & 0.09 \\
69 & 015332.17 & -741615.9 & 14.32 & 0.01 & 14.31 & 0.01 & 14.20 & 0.02 \\
70 & 015337.83 & -741713.4 & 16.26 & 0.02 & 16.19 & 0.03 & 16.01 & 0.06 \\
71 & 015337.93 & -743951.2 & 16.32 & 0.02 & 16.28 & 0.02 & 16.18 & 0.04 \\
72 & 015344.83 & -741318.2 & 15.66 & 0.02 & 15.58 & 0.02 & 15.29 & 0.03 \\
73 & 015346.73 & -741622.7 & 16.76 & 0.02 & 16.81 & 0.06 & 16.69 & 0.10 \\
74 & 015349.07 & -735916.3 & 16.48 & 0.02 & 16.40 & 0.02 & 16.23 & 0.05 \\
75 & 015349.85 & -735656.1 & 14.46 & 0.02 & 14.34 & 0.02 & 14.08 & 0.02 \\
76 & 015350.50 & -744340.0 & 16.61 & 0.02 & 16.55 & 0.03 & 16.43 & 0.06 \\
77 & 015410.53 & -735858.5 & 17.22 & 0.05 & 17.16 & 0.03 & 16.99 & 0.08 \\
78 & 015413.38 & -745123.6 & 16.84 & 0.03 & 16.76 & 0.04 & 16.67 & 0.08 \\
79 & 015414.81 & -735512.5 & 17.33 & 0.02 & 17.36 & 0.04 & 17.21 & 0.10 \\
80 & 015418.50 & -743423.9 & 16.51 & 0.02 & 16.41 & 0.03 & 16.29 & 0.05 \\
81 & 015443.21 & -740136.5 & 17.07 & 0.02 & 17.07 & 0.04 & 16.94 & 0.10 \\
82 & 015443.69 & -740013.2 & 17.16 & 0.03 & 17.21 & 0.04 & 17.06 & 0.09 \\
83 & 015449.63 & -741238.5 & 16.24 & 0.02 & 16.04 & 0.09 & 15.79 & 0.04 \\
84 & 015451.65 & -740150.2 & 15.83 & 0.01 & 15.80 & 0.02 & 15.65 & 0.04 \\
85 & 015451.73 & -741840.8 & 16.00 & 0.02 & 15.90 & 0.02 & 15.73 & 0.04 \\
86 & 015457.60 & -743757.9 & 16.71 & 0.02 & 16.70 & 0.04 & 16.50 & 0.06 \\
87 & 015506.93 & -740924.5 & 16.49 & 0.02 & 16.43 & 0.02 & 16.27 & 0.05 \\
88 & 015530.19 & -743814.1 & 16.27 & 0.01 & 16.24 & 0.03 & 16.13 & 0.05 \\
89 & 015535.87 & -735822.6 & 15.09 & 0.02 & 14.99 & 0.01 & 14.77 & 0.02 \\
90 & 015537.87 & -744643.2 & 15.18 & 0.02 & 15.07 & 0.02 & 14.84 & 0.03 \\
91 & 015547.27 & -741055.1 & 16.86 & 0.02 & 16.73 & 0.05 & 16.37 & 0.05 \\
92 & 015553.77 & -741109.1 & 16.03 & 0.03 & 15.94 & 0.02 & 15.80 & 0.04 \\
93 & 015555.01 & -740030.6 & 14.71 & 0.02 & 14.51 & 0.02 & 14.31 & 0.02
\end{tabular}


Table 1-Continued

\begin{tabular}{rrrrrrrrr}
\hline \hline ID & R.A. (J2000.0) & Dec. (J2000.0) & $J$ & $\sigma_{J}$ & $H$ & $\sigma_{H}$ & $K_{S}$ & $\sigma_{K_{S}}$ \\
\hline 94 & 015602.23 & -734045.2 & 17.29 & 0.03 & 17.22 & 0.05 & 16.93 & 0.08 \\
95 & 015608.95 & -740819.8 & 15.37 & 0.02 & 15.23 & 0.02 & 15.00 & 0.03 \\
96 & 015622.95 & -741818.1 & 16.34 & 0.03 & 16.25 & 0.04 & 16.04 & 0.07 \\
97 & 015631.73 & -735450.9 & 16.94 & 0.03 & 16.91 & 0.04 & 16.67 & 0.07 \\
98 & 015639.51 & -741255.4 & 13.98 & 0.02 & 14.01 & 0.02 & 13.86 & 0.02 \\
99 & 015642.99 & -741303.6 & 16.13 & 0.02 & 16.12 & 0.02 & 15.89 & 0.05 \\
100 & 015645.11 & -741305.4 & 16.77 & 0.03 & 16.83 & 0.04 & 16.67 & 0.06 \\
101 & 015646.59 & -741314.6 & 16.56 & 0.02 & 16.55 & 0.04 & 16.27 & 0.07 \\
102 & 015649.79 & -741335.3 & 16.45 & 0.02 & 16.37 & 0.03 & 16.03 & 0.05 \\
103 & 015651.73 & -741301.8 & 16.79 & 0.02 & 16.91 & 0.04 & 16.82 & 0.08 \\
104 & 015651.77 & -734612.8 & 15.84 & 0.02 & 15.80 & 0.02 & 15.64 & 0.03 \\
105 & 015652.50 & -733925.7 & 15.90 & 0.01 & 15.84 & 0.02 & 15.76 & 0.04 \\
106 & 015658.74 & -741651.3 & 15.59 & 0.02 & 15.49 & 0.02 & 15.26 & 0.03 \\
107 & 015703.55 & -734616.0 & 17.09 & 0.02 & 16.98 & 0.05 & 16.77 & 0.09 \\
108 & 015710.58 & -740549.1 & 16.05 & 0.02 & 16.04 & 0.02 & 15.88 & 0.04 \\
109 & 015735.09 & -741937.1 & 16.53 & 0.02 & 16.47 & 0.03 & 16.32 & 0.04 \\
110 & 015745.02 & -740000.4 & 15.14 & 0.02 & 15.04 & 0.02 & 14.87 & 0.03 \\
111 & 015751.29 & -742024.8 & 16.50 & 0.02 & 16.54 & 0.04 & 16.44 & 0.06 \\
112 & 015844.88 & -735323.1 & 15.19 & 0.02 & 15.05 & 0.02 & 14.77 & 0.02 \\
113 & 015901.43 & -742415.9 & 15.14 & 0.01 & 14.99 & 0.02 & 14.84 & 0.02 \\
114 & 015903.50 & -743024.9 & 14.67 & 0.02 & 14.57 & 0.02 & 14.37 & 0.03 \\
115 & 015925.89 & -741528.0 & 14.78 & 0.01 & 14.65 & 0.02 & 14.38 & 0.02 \\
116 & 015932.05 & -735608.2 & 15.24 & 0.02 & 15.08 & 0.02 & 14.87 & 0.02 \\
117 & 015940.95 & -742706.0 & 16.27 & 0.02 & 16.26 & 0.04 & 16.12 & 0.09 \\
118 & 015949.34 & -742348.0 & 17.35 & 0.03 & 17.30 & 0.08 & 17.13 & 0.10 \\
119 & 020003.41 & -740624.3 & 16.05 & 0.02 & 16.05 & 0.04 & 15.80 & 0.07 \\
120 & 020031.97 & -745038.1 & 16.64 & 0.02 & 16.58 & 0.03 & 16.42 & 0.10 \\
121 & 020102.89 & -735548.5 & 17.20 & 0.02 & 17.18 & 0.05 & 17.05 & 0.09 \\
122 & 020109.38 & -734037.9 & 16.35 & 0.02 & 16.21 & 0.04 & 16.09 & 0.10 \\
123 & 020126.95 & -744014.8 & 15.21 & 0.02 & 15.15 & 0.02 & 14.97 & 0.03 \\
124 & 020133.99 & -740909.4 & 16.36 & 0.02 & 16.29 & 0.03 & 16.08 & 0.05
\end{tabular}


Table 1-Continued

\begin{tabular}{ccccccccc}
\hline \hline ID & R.A. (J2000.0) & Dec. (J2000.0) & $J$ & $\sigma_{J}$ & $H$ & $\sigma_{H}$ & $K_{S}$ & $\sigma_{K_{S}}$ \\
\hline 125 & 020159.96 & -745156.6 & 16.10 & 0.01 & 15.94 & 0.03 & 15.59 & 0.05 \\
126 & 020309.38 & -735527.6 & 16.91 & 0.02 & 16.72 & 0.05 & 16.51 & 0.08 \\
127 & 020311.72 & -740334.5 & 17.28 & 0.02 & 17.20 & 0.05 & 17.05 & 0.09 \\
128 & 020317.45 & -733928.4 & 14.85 & 0.02 & 14.68 & 0.06 & 14.43 & 0.03 \\
129 & 020332.50 & -735833.7 & 17.00 & 0.03 & 17.00 & 0.07 & 16.78 & 0.09 \\
130 & 020333.40 & -740115.8 & 14.17 & 0.02 & 14.16 & 0.03 & 13.84 & 0.03 \\
131 & 020352.08 & -740659.8 & 15.22 & 0.02 & 15.04 & 0.04 & 14.92 & 0.02 \\
132 & 020419.90 & -744548.8 & 16.94 & 0.03 & 16.89 & 0.04 & 16.67 & 0.08 \\
133 & 020430.52 & -740751.5 & 16.31 & 0.02 & 16.15 & 0.03 & 16.04 & 0.04 \\
134 & 020440.50 & -745345.6 & 15.58 & 0.03 & 15.39 & 0.03 & 15.23 & 0.05 \\
135 & 020442.14 & -734648.2 & 15.65 & 0.02 & 15.55 & 0.02 & 15.34 & 0.03 \\
136 & 020458.60 & -743158.3 & 16.69 & 0.02 & 16.58 & 0.03 & 16.48 & 0.06 \\
137 & 020513.61 & -740825.0 & 15.42 & 0.02 & 15.23 & 0.02 & 15.03 & 0.05 \\
138 & 020515.55 & -741126.8 & 16.90 & 0.03 & 17.00 & 0.05 & 16.82 & 0.09 \\
139 & 020616.16 & -741051.1 & 16.96 & 0.03 & 17.08 & 0.06 & 16.97 & 0.10 \\
140 & 020621.48 & -743719.6 & 14.94 & 0.03 & 14.89 & 0.02 & 14.80 & 0.05 \\
141 & 020636.90 & -740538.3 & 17.11 & 0.03 & 17.10 & 0.05 & 16.85 & 0.09 \\
142 & 020641.33 & -742036.2 & 15.82 & 0.02 & 15.76 & 0.02 & 15.59 & 0.04 \\
143 & 020645.18 & -742747.5 & 14.96 & 0.01 & 14.93 & 0.02 & 14.79 & 0.02 \\
144 & 020647.75 & -742121.6 & 16.26 & 0.02 & 16.10 & 0.03 & 15.97 & 0.04 \\
145 & 020703.07 & -745337.6 & 17.40 & 0.04 & 17.22 & 0.05 & 16.97 & 0.10 \\
146 & 020711.53 & -744420.7 & 17.02 & 0.03 & 17.05 & 0.05 & 16.95 & 0.10 \\
147 & 020717.97 & -740936.0 & 16.26 & 0.02 & 16.20 & 0.04 & 15.90 & 0.05 \\
148 & 020732.23 & -750037.4 & 14.87 & 0.02 & 14.73 & 0.01 & 14.49 & 0.02 \\
149 & 020733.24 & -744748.7 & 14.97 & 0.01 & 14.92 & 0.01 & 14.77 & 0.03 \\
150 & 020733.27 & -740103.3 & 15.68 & 0.04 & 15.60 & 0.02 & 15.51 & 0.03 \\
151 & 020734.11 & -742008.5 & 13.99 & 0.02 & 14.00 & 0.01 & 13.89 & 0.02 \\
152 & 020737.06 & -743250.8 & 17.00 & 0.02 & 16.92 & 0.04 & 16.69 & 0.10 \\
153 & 020754.58 & -743325.4 & 16.32 & 0.02 & 16.28 & 0.03 & 16.11 & 0.07 \\
154 & 020806.80 & -741804.6 & 14.32 & 0.01 & 14.22 & 0.01 & 13.90 & 0.02 \\
155 & 020809.54 & -741915.3 & 15.89 & 0.02 & 15.83 & 0.02 & 15.61 & 0.03
\end{tabular}


Table 1-Continued

\begin{tabular}{|c|c|c|c|c|c|c|c|c|}
\hline ID & R.A. (J2000.0) & Dec. (J2000.0) & $J$ & $\sigma_{J}$ & $H$ & $\sigma_{H}$ & $K_{S}$ & $\sigma_{K_{S}}$ \\
\hline 156 & $0208 \quad 17.33$ & -742338.3 & 15.65 & 0.02 & 15.57 & 0.02 & 15.31 & 0.03 \\
\hline 157 & 020819.54 & & & 0.01 & 13.44 & 0.01 & & 0.02 \\
\hline 158 & 020821.16 & & & 0.03 & & 0.04 & & 0.09 \\
\hline 159 & 020824.08 & & 15.22 & 0.03 & & 0.02 & & 0.03 \\
\hline 160 & & & & 0.02 & 16.91 & 0.05 & 16.72 & .10 \\
\hline 161 & & & 17.03 & 0.03 & 16.98 & 0.07 & & 0.10 \\
\hline 162 & 020838.6 & & & 0.01 & 16.22 & 0.03 & & 0.05 \\
\hline 163 & & & & 0.02 & & 0.03 & & 0.09 \\
\hline 164 & 020900 & -73 & & 0.02 & 16.25 & 0.03 & 12 & 0.05 \\
\hline 165 & & & & 0.03 & 17.02 & 0.05 & & .08 \\
\hline 166 & 0 & & 15.63 & 0.01 & 15.55 & 0.02 & 15 & 0.08 \\
\hline 167 & 020916.01 & & & 0.02 & & 0.02 & & 0.05 \\
\hline 168 & 02092 & & & 0.02 & 16.46 & 0.04 & & 0.07 \\
\hline 169 & 020929 & -74 & 16.37 & 0.02 & 16.30 & 0.03 & 03 & 0.05 \\
\hline 170 & & & 16.71 & 0.02 & 16.71 & 0.06 & 47 & 0.09 \\
\hline 171 & & -7 & 14.35 & 0.02 & 14.27 & 0.02 & 14.10 & 0.02 \\
\hline 172 & & & 16.32 & 0.03 & 16.38 & 0.05 & 16.26 & 0.05 \\
\hline 173 & & & & 0.02 & 16.75 & 0.05 & 16. & 0.09 \\
\hline 174 & & & & & & 0.06 & 17. & 0.10 \\
\hline 175 & & & 14.51 & 0.03 & 14.32 & 0.04 & 14.17 & 0.02 \\
\hline 176 & 021045.27 & -74 & 15.76 & 0.01 & 15.71 & 0.02 & 15.46 & 0.03 \\
\hline 177 & & -735222.5 & 15.34 & 0.02 & 15.20 & 0.02 & 14.97 & 0.03 \\
\hline 178 & & & 15.83 & 0.02 & 15.95 & 0.02 & 15.84 & 0.04 \\
\hline 179 & & & & & & & & 0.09 \\
\hline 180 & & & & 0.02 & 16.68 & 0.04 & & 0.09 \\
\hline 181 & 021118.80 & -74295 & 14.31 & 0.06 & 14.23 & 0.06 & 13.85 & 0.08 \\
\hline 182 & 021120.67 & -734533.3 & 17.43 & 0.02 & 17.39 & 0.05 & 17.22 & 0.10 \\
\hline 183 & 021129.18 & & & 0.02 & 16.56 & 0.03 & 16.36 & 0.06 \\
\hline 184 & & & & 0.03 & & 0.06 & & 0.02 \\
\hline 185 & 021138.26 & & 16.82 & 0.02 & 16.70 & 0.04 & 16.50 & 0.06 \\
\hline 186 & 021143.72 & -741738.6 & 14.23 & 0.01 & 14.18 & 0.01 & 14.02 & 0.02 \\
\hline
\end{tabular}




\section{REFERENCES}

Ahumada, A. V., Clariá J. J., Bica, E., \& Dutra, C. M. 2002, A\&A, 393, 855

Bernasconi, P. A., 1996, A\&AS, 120, 57

Bernasconi, P. A., \& Maeder, A. 1996, A\&A, 307, 829

Bica, E. L. D., \& Schmitt, H. R. 1995, ApJ, 104, 41

Beaulieu, J. P., et al. 1996, Science, 272, 995

Beaulieu, J. P., et al. 2001, A\&A, 380, 168

Bohlin, R. C., Savage, B. D., \& Drake, J. F. 1978, ApJ, 224, 132

Brüns, C., Kerp, J., Staveley-Smith, L., Mebold, U., Putman, M. E., Haynes, R. F., Kalberla, P. M. W., Muller, E., \& Filipovic, M. D. 2005, A\&A, 432, 45

Demers, S., \& Battinelli, P. 1998, AJ, 115, 154

de Wit, W. J., Beaulieu, J. P., \& Lamers, H. J. G. L. M. 2002, A\&A, 395, 829

de Wit, W. J., Beaulieu, J. P., Lamers, H. J. G. L. M., Lesquoy, E., \& Marquette, J. B. 2003, A\&A, 410, 199

de Wit, W. J., Beaulieu, J. P., Lamers, H. J. G. L. M., Coutures, C., \& Meeus, G. 2005, A\&A, 432, 619

Fabregat. J., \& Torrejón. J. M. 2000, A\&A, 357, 451

Gardiner, L. T., Sawa, T., \& Fujimoto, M. 1994, MNRAS, 266, 567

Grondin, L., \& Demers, S. 1993, in ASP Conf. Ser. 45, Luminous High-Latitude Stars, ed. D. D. Sasselov (San Francisco: ASP), 380

Harries, T. J., Hilditch, R. W., \& Howarth, I. D. 2003, MNRAS, 339, 157

Hillenbrand, L. A., Strom, S. E., Vrba, F. J., \& Keene, J. 1992, ApJ, 397, 613

Hindman, J. V., Kerr, F. J., \& McGee, R. X. 1963, AuJPh, 16, 570

Irwin, M. J., Demers, S., \& Kunkel, W. E. 1990, AJ, 99, 191

Keller, S. C., Wood, P. R., \& Bessell, M. S. 1999, A\&AS, 134, 489 
Table 1-Continued

\begin{tabular}{ccccccccc}
\hline \hline ID & R.A. (J2000.0) & Dec. (J2000.0) & $J$ & $\sigma_{J}$ & $H$ & $\sigma_{H}$ & $K_{S}$ & $\sigma_{K_{S}}$ \\
\hline 187 & 021225.79 & -742235.2 & 15.41 & 0.02 & 15.34 & 0.02 & 15.25 & 0.03 \\
188 & 021235.51 & -734216.8 & 17.20 & 0.03 & 17.01 & 0.05 & 16.83 & 0.06 \\
189 & 021238.38 & -742244.9 & 16.23 & 0.02 & 16.23 & 0.03 & 16.08 & 0.06 \\
190 & 021239.40 & -745751.1 & 15.71 & 0.02 & 15.63 & 0.02 & 15.50 & 0.03 \\
191 & 021258.10 & -745428.4 & 15.08 & 0.02 & 14.99 & 0.03 & 14.60 & 0.02 \\
192 & 021358.31 & -740648.2 & 16.17 & 0.03 & 16.28 & 0.04 & 16.18 & 0.05 \\
193 & 021412.83 & -744010.4 & 14.73 & 0.01 & 14.58 & 0.02 & 14.26 & 0.03 \\
194 & 021440.19 & -741205.7 & 15.85 & 0.03 & 15.74 & 0.02 & 15.49 & 0.04 \\
195 & 021442.36 & -741212.0 & 15.38 & 0.03 & 15.24 & 0.02 & 14.96 & 0.03 \\
196 & 021445.26 & -740744.6 & 16.12 & 0.03 & 16.05 & 0.03 & 15.74 & 0.04 \\
197 & 021532.54 & -740751.8 & 15.45 & 0.03 & 15.36 & 0.02 & 15.26 & 0.03 \\
198 & 021553.19 & -742638.7 & 15.75 & 0.03 & 15.56 & 0.09 & 15.34 & 0.03 \\
199 & 021601.20 & -740830.4 & 15.78 & 0.03 & 15.63 & 0.03 & 15.38 & 0.03 \\
200 & 021643.89 & -740004.9 & 14.96 & 0.02 & 14.88 & 0.03 & 14.54 & 0.03 \\
201 & 021651.67 & -741333.0 & 14.88 & 0.03 & 14.83 & 0.06 & 14.53 & 0.05 \\
202 & 021702.55 & -734007.5 & 14.83 & 0.03 & 14.72 & 0.08 & 14.40 & 0.03 \\
203 & 021743.06 & -742021.2 & 15.74 & 0.01 & 15.65 & 0.02 & 15.42 & 0.04 \\
\hline
\end{tabular}


Lamers, H. J. G. L. M., Beaulieu, J. P., \& de Wit, W. J. 1999, A\&A, 341, 827

McSwain, M. V., \& Gies, D. R. 2005, ApJS, 161, 118

Mizuno, N., Muller, E., Maeda, H., Kawamura, A., Minamidani, T., Onishi, T., Mizuno, A., \& Fukui, Y. 2006, ApJ, 643, L107

Muller, E., Staveley-Smith, L., Zealey, W., \& Stanimirović, S. 2003a, MNRAS, 339, 105

Muller, E., Staveley-Smith, L., \& Zealey, W. J. 2003b, MNRAS, 338, 609

Nagashima, C., et al. 1999, in Star Formation 1999, ed. T. Nakamoto (Nobeyama : Nobeyama Radio Obs.), 397

Nagayama, T., et al. 2003, Proc. SPIE, 4841, 459

Nakajima, Y., et al. 2005, AJ, 129, 776

Palla, F., \& Stahler, S. W. 1993, ApJ, 418, 414

Panagia, N., Romaniello, M., Scuderi, S., \& Kirshner, R. P. 2000, ApJ, 539, 197

Persson, S. E., Murphy, D. C., Krzeminski, W., Roth, M., \& Rieke, M. J. 1998, AJ, 116, 2475

Rolleston, W. R. J., Dufton, P. L., McErlean, N. D., \& Venn, K. A. 1999 A\&A, 348, 728

Romaniello, M., Robberto, M., \& Panagia, N. 2004, ApJ, 608, 220

Shapley, H. 1940, Harvard College Observatory Bulletin, 914, 8

Stetson, P. B. 1987, PASP, 99, 191

Tokunaga, A. T. 2000, Astrophysical Quantities, 4th edition, ed. Cox, A. (AIP Press), p.143

van de Hulst, H. C., 1946, Rech.Astr.Obs.Utrecht, 11, 1

Westerlund, B. E., \& Glaspey, J. 1971, A\&A, 10, 1

Yoshizawa, A. M., \& Noguchi, M., 2003, MNRAS, 339, 1135

Zorec, J., \& Briot, D. 1997, A\&A, 318, 443

Zhang, P., Chen, P. S., \& Yang, H. T. 2005, New A, 10, 325 
Fig. 1. - see figure1.jpg - Herbig Ae/Be candidates (crosses) within the western Magellanic Bridge (the wing of the SMC) found in this survey overlaid on an integrated H I intensity map (Muller et al. 2003a). Our observed area is shown by solid lines. The gray-scale is a linear transfer function, as shown on the intensity wedge with units in $\mathrm{K} \mathrm{km} \mathrm{s}^{-1}$. The position of the star cluster NGC $796\left(01^{\mathrm{h}} 56^{\mathrm{m}},-74^{\circ} 13^{\prime}\right)$ is shown by a circle.

Fig. 2.- see figure2.jpg $-J-H$ versus $H-K_{S}$ color-color diagram for the point sources detected in all bands with photometric error less than 0.1 mag (gray-scale). Crosses represent Herbig Ae/Be candidates. The thin and thick curves are the loci of dwarfs and giants, respectively (Tokunaga 2000). Their colors are transformed to the IRSF system with the color equations of Nakajima et al. (2005). The stars in the region labeled A are used to estimate the contamination by AF dwarfs. The three circles are 0.1 mag radius error circles centered at (a) B9, (b) B3, and (c) O6 dwarfs, which are used in the third criterion to select Herbig Ae/Be candidates (see text, Sec. 3). 


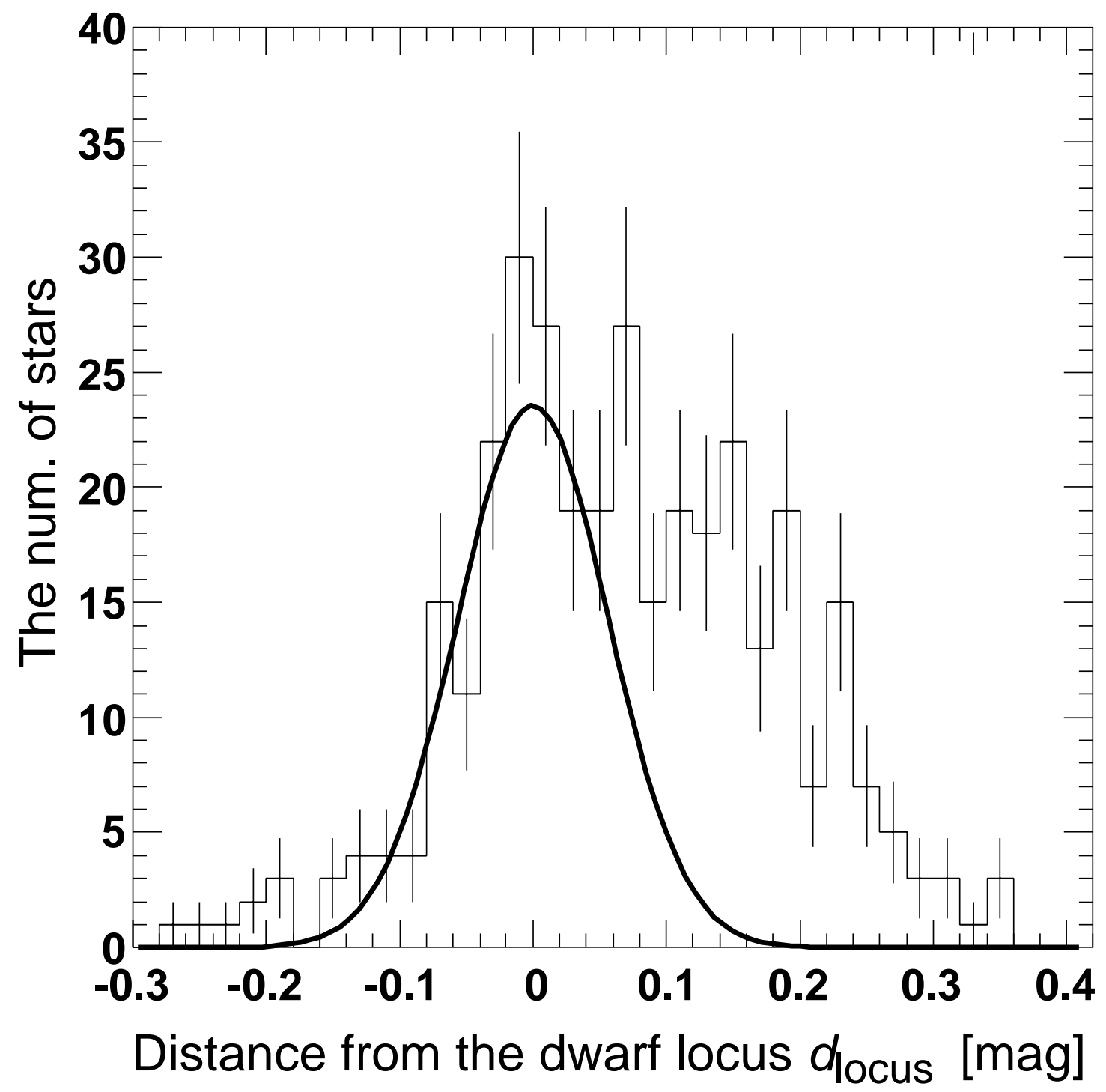

Fig. 3.- Distribution of distance from the dwarf A0-F5 locus for the point sources in the A region shown in Fig. 2. The dwarf locus is assumed to be a linear line with $J-H=$ $4.2 \times\left(H-K_{S}\right)-0.02$. The thick curve denotes a Gaussian distribution with a mean of 0 and sigma of 0.057 , which is derived by fitting the histogram at $d_{\text {locus }} \leq 0$. 


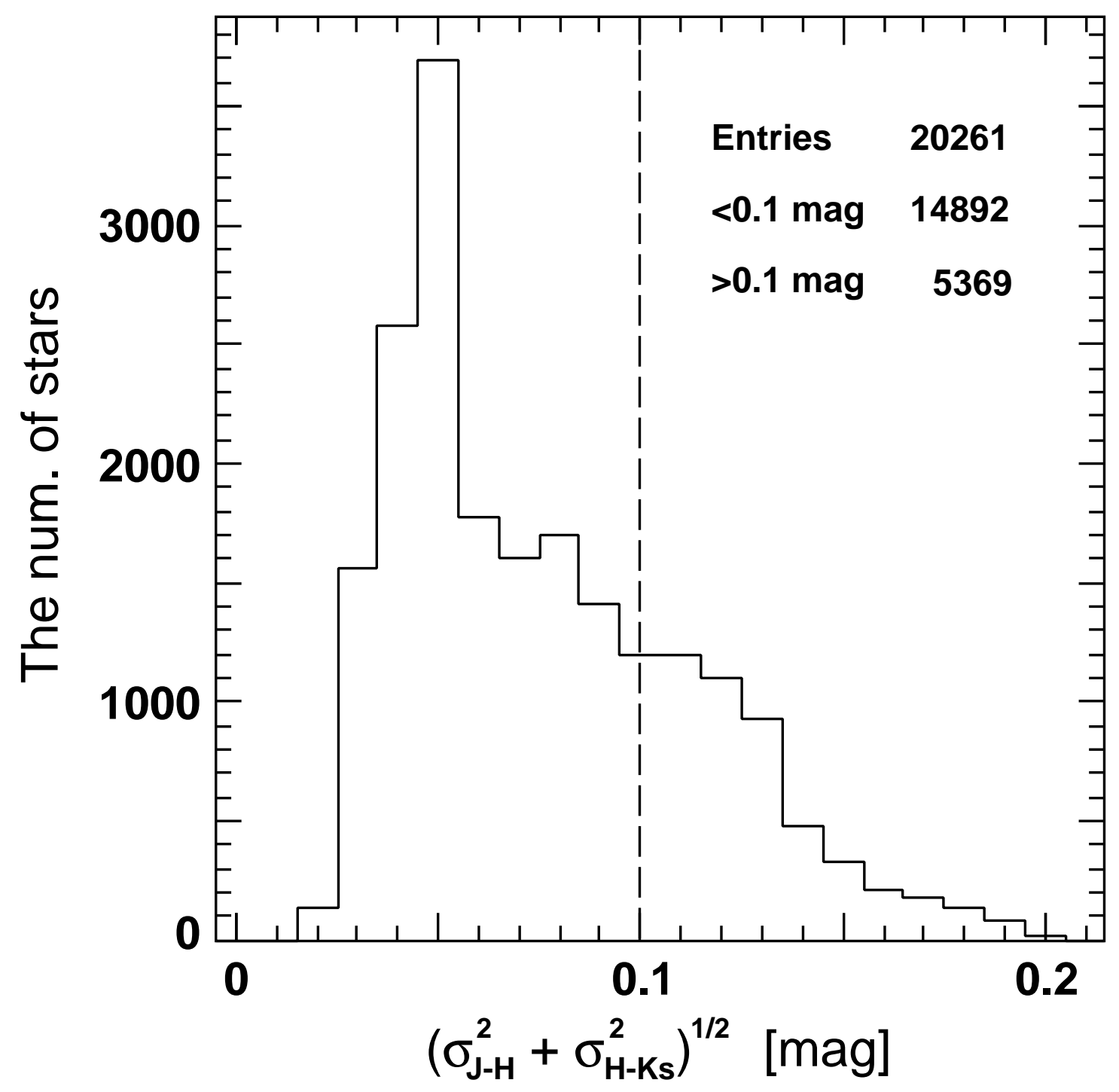

Fig. 4.- Histogram of $\sigma_{\text {tot }}=\sqrt{\sigma_{J-H}^{2}+\sigma_{H-K_{S}}^{2}}$ for all sources detected in the three bands with $\sigma_{J, H, K_{S}} \leq 0.1 \mathrm{mag}$. The numbers of sources with $\sigma_{\text {tot }}<0.1$ and $\sigma_{\text {tot }}>0.1 \mathrm{mag}$ are $14,892(74 \%)$ and $5,369(26 \%)$, respectively. 


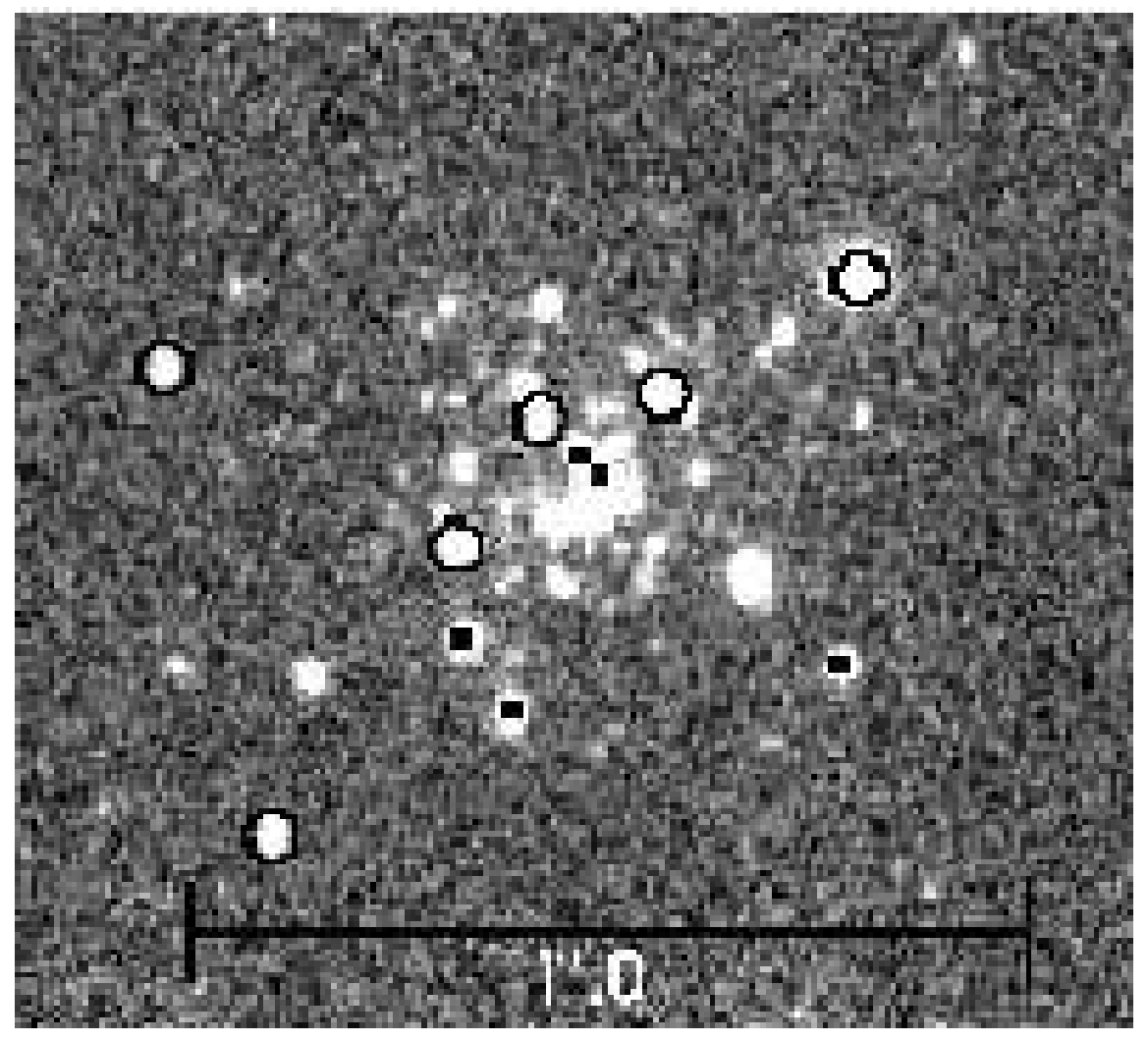

Fig. 5.- $J$ band image of NGC 796. A scale of $1^{\prime}$ is shown in the image. North is up, and east is to the left. Circles and squares represent Herbig Ae/Be star candidates and stars near the OB locus, respectively.

Fig. 6. - see figure6.jpg $-J$ versus $J-K_{S}$ color-magnitude diagram for point sources detected in all bands, with photometric error less than 0.1 mag (gray-scale). Crosses represent Herbig Ae/Be candidates found in our survey, and triangles and squares represent the Herbig Ae/Be candidates found in the LMC (de Wit et al. 2005) and in the SMC (de Wit et al. 2003), respectively. The solid curve is the locus of dwarfs (Tokunaga 2000). The dotted and dashed lines denote the masses of the group I/II and the group III Herbig Ae/Be stars, respectively, estimated by the empirical relationship between $J$ magnitude and stellar mass (Hillenbrand et al. 1992; Nakajima et al. 2005) at the distance of the MB (60 kpc). Their colors are transformed to the IRSF system with the color equations of Nakajima et al. (2005). 
This figure "figure1.jpg" is available in "jpg" format from: http://arxiv.org/ps/astro-ph/0702504v1 
This figure "figure2.jpg" is available in "jpg" format from: http://arxiv.org/ps/astro-ph/0702504v1 
This figure "figure6.jpg" is available in "jpg" format from: http://arxiv.org/ps/astro-ph/0702504v1 